\title{
Comparison of the Window-Frame RHIC-abort kicker with C-type Kicker
}

\author{
N. Tsoupas, H. Hahn, \\ M. Severance, B. McMahan
}

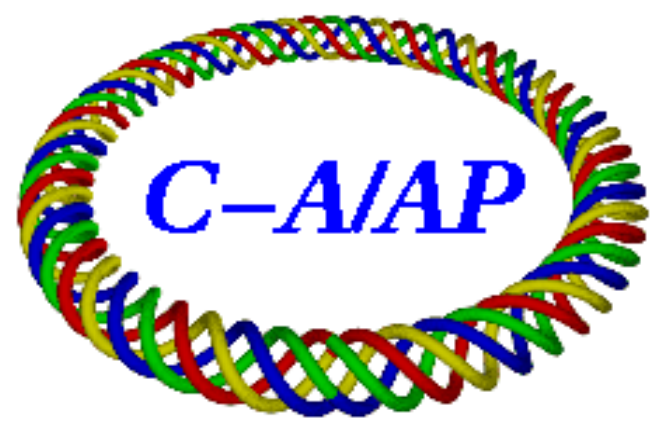

\section{Collider-AcceleratorDepartment Brookhaven National Laboratory Upton, NY 11973}

\section{U.S. Department of Energy Office of Science, Office of Nuclear Physics}

Notice: This document has been authorized by employees of Brookhaven Science Associates, LLC under Contract No. DE-AC02-98CH10886 with the U.S. Department of Energy. The United States Government retains a nonexclusive, paid-up, irrevocable, world-wide license to publish or reproduce the published form of this document, or allow others to do so, for United States Government purposes. 


\title{
Comparison of the Window-Frame RHIC-abort kicker with C-type Kicker
}

\author{
N. Tsoupas, H. Hahn, W. Meng, BNL \\ Michael Severance, Stony Brook University \\ Brandon McMahan, Westhampton High School
}

\begin{abstract}
The high intensity proton bunches $\left(\sim 2.5 \times 10^{11} \mathrm{p} /\right.$ bunch $)$ circulating in RHIC increase the temperature of the ferrite-made RHIC-abort-kickers above the Curie point, as a result the kickers cannot provide the required field to abort the beam at the beam dump. A team of experts in the CAD department worked on modifying the design of the window-frame RHIC-abort kicker to minimize the hysteresis losses responsible for the increase of the ferrite's temperature. In this technical note we report some results from the study of two possible modifications of the window-frame RHIC-abort kicker and we compare these results with those of a propose C-type RHIC-abort kicker. We also include an Appendix where we describe a method which may further reduce the hysteresis losses of the window-frame kicker.
\end{abstract}

\section{Introduction}

During the polarized proton run-2013 the RHIC-abort kickers failed few times to provide the required B_field to abort the circulating RHIC bunches to the beam dump made of carbon fiber. Theoretical calculations and experimental studies attribute this failure to the temperature increase of the CMD5005 ferrite above the Curie point, and concluded that this temperature increase is due to the hysteresis losses caused by the time varying magnetic field induced in the ferrite by the relatively high beam current of $\sim 2.0 \times 10^{11} \mathrm{p} /$ bunch of the circulating proton beam bunches. A team of experts in the CAD department worked on modifying the design of the window-frame RHIC-abort kicker to minimize the hysteresis losses responsible for the increase of the ferrite's temperature. In this technical note we provide results from the theoretical study performed on two possible designs of the window-frame kickers which minimize the hysteresis losses in the ferrite material, and we compare the performance of these two window-frame kickers with a proposed C-type kicker. The calculations were performed using the "ac" and the "transient" modules of the 2D OPERA computer code.

\section{The Window-frame kicker}

In this section we describe the modeling of the window-frame RHIC-abort-kicker and the results from the beam induced hysteresis and ohmic losses in the kicker. The modeling of the kicker and the calculations were performed by the use of the "ac-module" of the 2D OPERA computer code. Figure 1 shows a vertical cross section of the RHIC-abort kicker. The green thin ring is the stainless steel vacuum pipe and the blue shaded area corresponds to the ferrite of the kicker. The aqua-marine thin vertical rectangles are the current carrying conductors which are made of stainless steel. The red circle in the center of the kicker shows the beam current which is simulated as a sinusoidal function with amplitude of $1.5 \mathrm{~A}$ and frequency 10 [MHz]. The two thin brown vertical lines inside the return yolk of the ferrite are 2 [mm] 
thick copper strips running along the length of the kicker to minimize the $\mathrm{H}$ _field induced in the ferrite of the kicker caused by the circulating beam bunches. The conductivity for the stainless steel, copper, and ferrite used in the model are $\sigma_{\mathrm{ss}}=1.4204 \times 10^{4}(\Omega \mathrm{m})^{-1}, \sigma_{\mathrm{co}}=5.78 \times 10^{7}(\Omega \mathrm{m})^{-1}$, and $\sigma_{\mathrm{fe}}=1 \times 10^{-9}(\Omega \mathrm{m})^{-1}$. The simulation of the hysteresis losses in the ferrite of the kicker is described briefly in the subsection below.

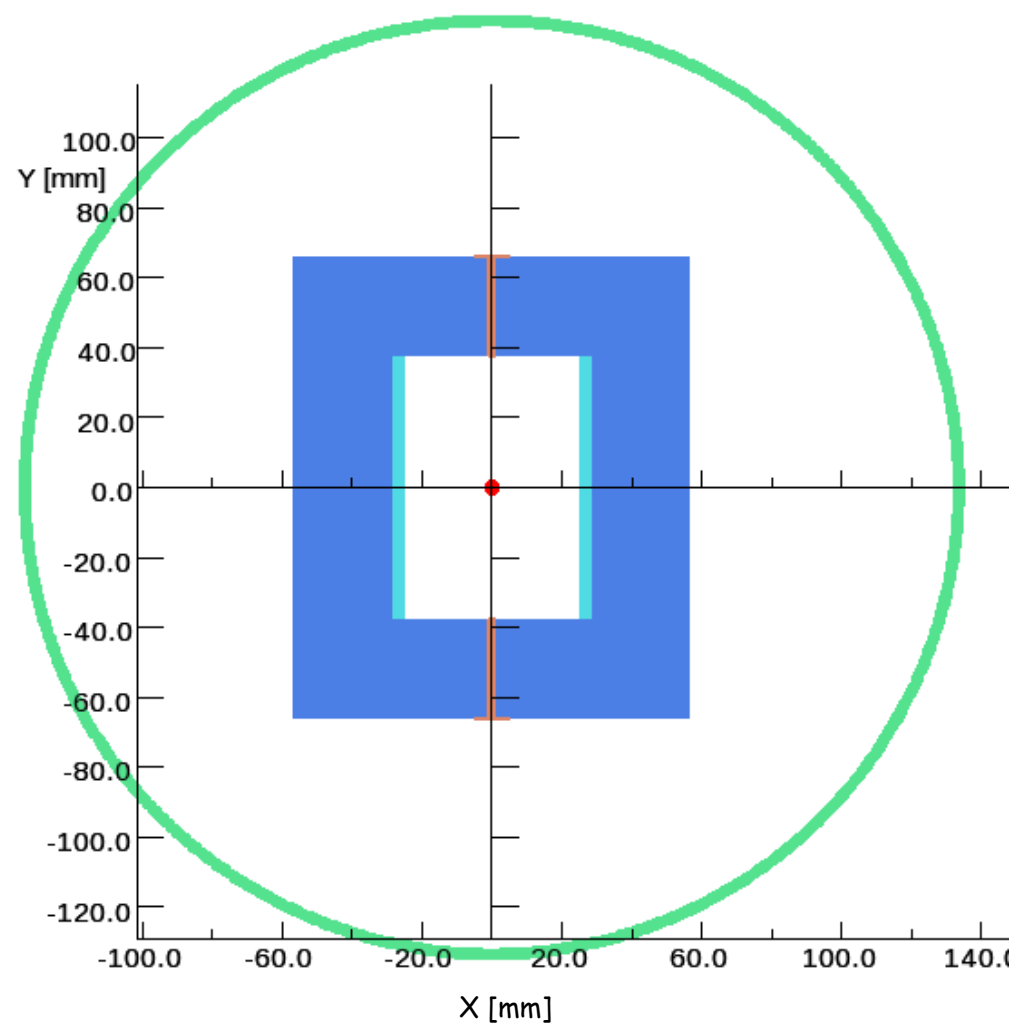

Figure 1. A vertical cross section of the window-frame RHIC-abort kicker. The thin green ring is the stainless steel vacuum pipe and the blue colored area corresponds to the ferrite material. The vertical aqua-marine rectangles are the current carrying conductors which are made of stainless steel. The red circle in the center of the magnet shows the beam current which is simulated as a sinusoidal function with amplitude of 1.5 [A] and frequency 10 [MHz]. The thin brown areas are 2 [mm] thick copper strips running along the length of the kicker to minimize the H_field induced in the ferrite by the beam current.

\section{Modeling of the hysteresis losses}

We assume that the current generated by the beam bunches is equivalent to a sinusoidal current with amplitude 1.5 [A] and frequency $10 \mathrm{MHz}$. The beam current is running in the center of the kicker magnet. Although this is not an accurate representation of the current generated by the 120 circulating beam bunches of $\sim 5$ nsec long containing $\sim 2.0 \times 10^{11}$ protons each, it is one way that we can calculate the hysteresis losses in the ferrite by using the "ac" module of the 2D opera code. Also our main task in this technical note is to compare the hysteresis losses between the two window-frame kickers and with the proposed C-type kicker, rather than provide an accurate number of the hysteresis losses for each kicker. This alternating beam current generates in the ferrite an $\mathrm{H}$ _field which can be expressed as $\mathrm{H}(\mathrm{t})=\mathrm{H}_{0} \sin (\omega \mathrm{t})$. If the corresponding $\mathrm{B}$ _field generated in the ferrite is also given by $\mathrm{B}(\mathrm{t})=\mathrm{B}_{0} \sin (\omega \mathrm{t})$ it is 
easy to calculate that the hysteresis losses $\int_{0}^{T} H(t) d B(t)$ per period are equal to zero. The experimentally measured increase of the temperature on various locations on the surface of the ferrite was too high to be attributed to eddy current losses in the ferrite because of the low electrical conductivity $\sigma=1 \times 10^{-9}(\Omega \mathrm{m})^{-1}$ of the ferrite. Therefore the temperature increase is due to hysteresis losses. The hysteresis losses are due to the lag of the B_field vector with respect to H_field one. The B_field in the ferrite can be expressed therefore as a sine-function $\mathrm{B}(\mathrm{t})=\mathrm{B}_{0} \sin (\omega \mathrm{t}-\delta)=\mathrm{B}_{0}[\sin (\omega \mathrm{t}) \cos \delta$ - $\cos (\omega \mathrm{t}) \sin \delta]$. Thus the hysteresis losses per period are given by the formula below.

$\int_{0}^{T} H(t) d B(t)=\int_{0}^{T} H_{0} \sin (\omega t)\left(B_{0} \omega\right) \cos (\omega t) \cos \delta d t+\int_{0}^{T} H_{0} \cos (\omega t)\left(B_{0} \omega\right) \cos (\omega t) \sin \delta d t=\frac{1}{2} H_{0} B_{0} \sin \delta$ (1) Figure 2 shows the physical meaning of the integral $\int_{0}^{T} H(t) d B(t)$ as the area enclosed by the hysteresis curve depicted by the thick blue line. The area enclosed by this curve represents the hysteresis losses. From equation (1) it is apparent that the "hysteresis losses" in the ferrite are caused by the lag-angle $\delta$ of the B_field relative to the H_field. Also from equation (1) the minimization of the hysteresis losses can be achieved by minimizing the H_field in the ferrite. To minimize the H_field in the ferrite, copper strips, shown by thin vertical brown lines in Figure 1, were inserted along the length of the ferrite. These copper strips generate eddy currents that create H_field which oppose the H_field generated by the circulating beam bunches.

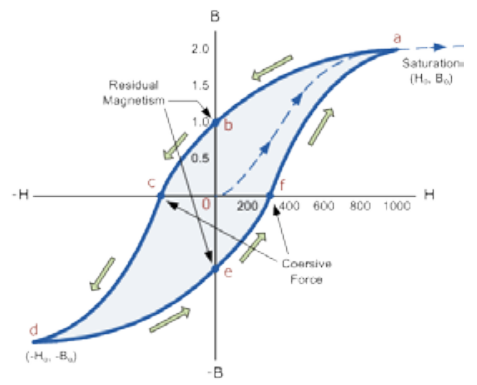

Figure 2. A typical hysteresis curve shown by the thick blue line. The area enclosed by the hysteresis curve corresponds to the "hysteresis losses".

Figure 3 shows graphically how the reduction of the H_field reduces the area of the hysteresis loops therefore minimizing the hysteresis losses.

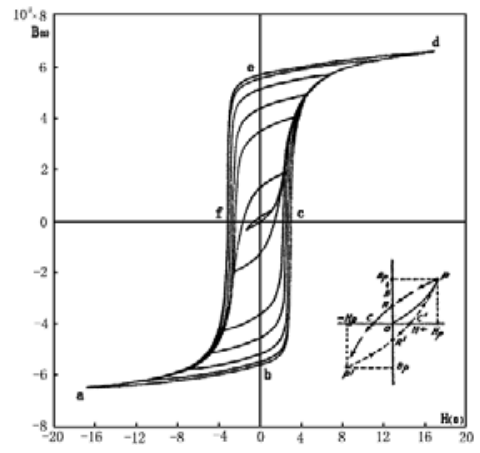

Figure 3. Hysteresis loops for various strengths of applied H_fields. The plots on this graph shows the reduction of the areas of the hysteresis loops with the reduction of the maximum value of the H_field. 


\section{Results from the calculations on the window-frame kickers}

Figures 4a and 4b show the two possible models of the window-frame RHIC-abort kicker, one of which will be used in RHIC. The difference of these two models is in the region of the copper strips, as can be realized by simply comparing Figures $4 \mathrm{a}$ and $4 \mathrm{~b}$.

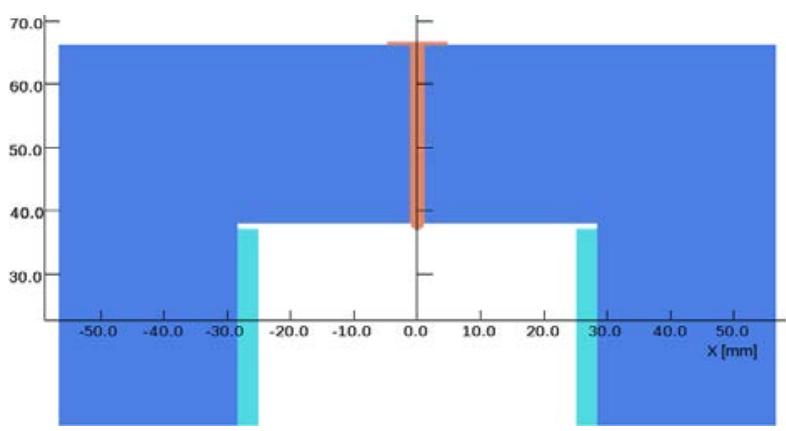

(a)

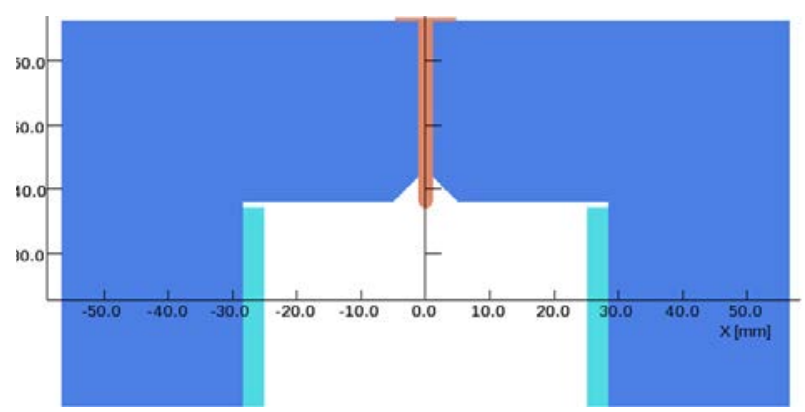

(b)

Figure 4a and 4b. The upper part of the cross section of the two possible models of the window-frame kicker to be used as RHIC-abort kickers. The comparison of the models shown in Figures 4a and 4b depicts the difference between the two possible models, one of which will be used as RHIC-abort kicker. The horizontal and vertical scales are in mm.

The BH curve used in the calculations is shown in Figure 5, and the lag-angle $\delta$ of the ferrite material used in the calculations was set equal to $45^{\circ}$.

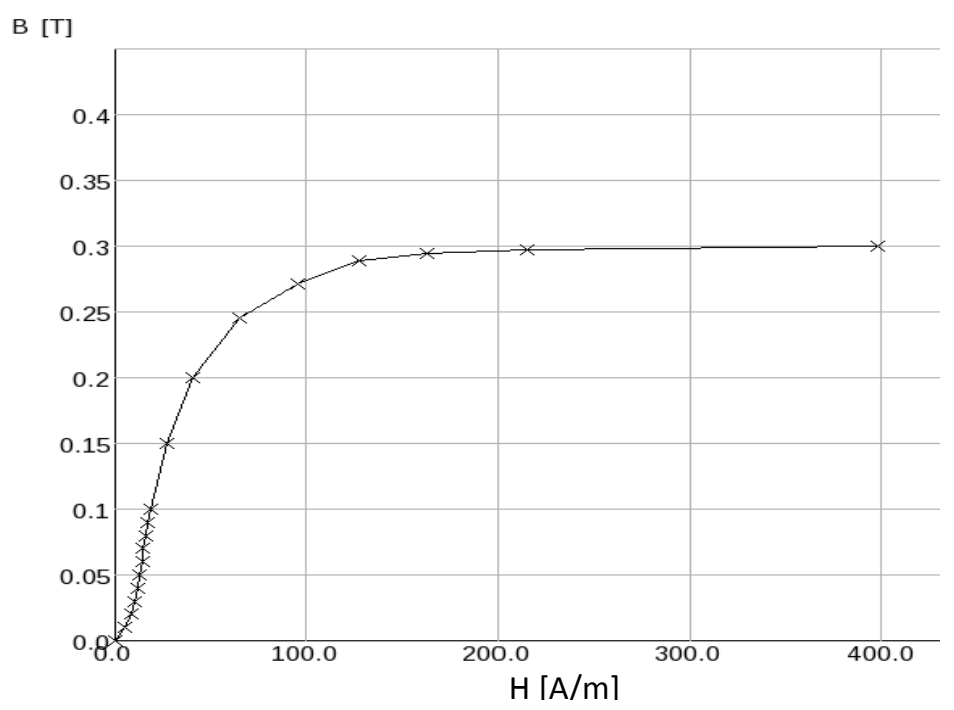

Figure 5. The BH curve of the CMC5005 ferrite material which was used in the 2D OPERA calculations.

The yellow lines in Figure 6 correspond to the vector potential lines generated by the "ac-module" of the 2D OPERA computer model. Note that the copper strips prevent the field lines to pass through the strips because an opposing H_field is generated by the eddy currents in the copper strips. 


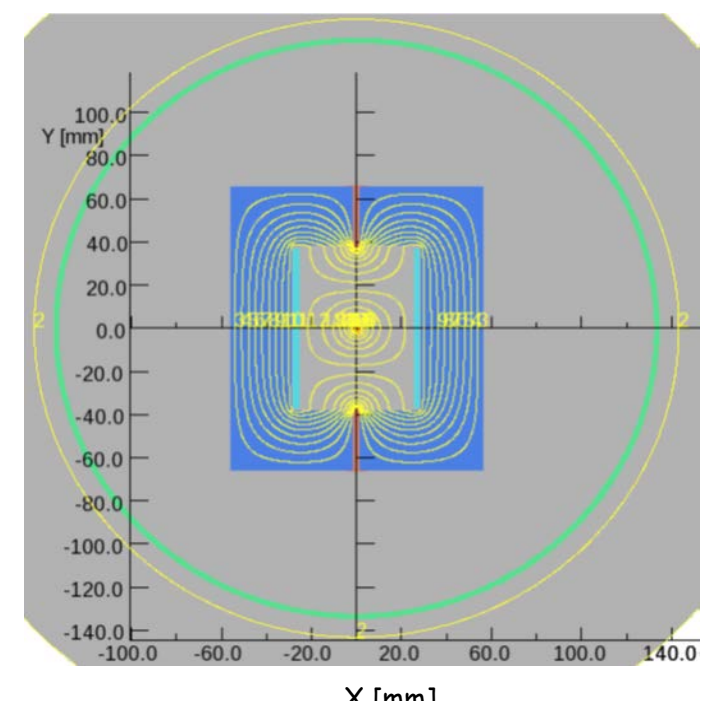

Figure 6. The yellow lines are the vector potential lines generated by the "ac-module" of the 2D OPERA computer model. Note that the copper strips prevent the field lines to pass through them because the eddy currents generated in the copper strips generate an $\mathrm{H} \_$field which opposes the $\mathrm{H}$ _field generated by the beam current. The horizontal and vertical scales are in $\mathrm{mm}$.

The hysteresis and the eddy current losses in the various parts of each of the two models are shown in lines 2,3, and 4 of Table 1 . The $2^{\text {nd }}$ column in Table 1 shows the amplitude of the sinusoidal beam current, and the rest of the columns show the hysteresis and eddy-current (ohmic) losses in the various materials of the kicker. The calculations show that the window-frame kicker of Figure $4 \mathrm{~b}$ generates $4.5 \%$ less hysteresis losses as compared to the hysteresis losses window-frame kicker shown in Figure 4a. Line 5 in Table 1 shows that the hysteresis and eddy-current losses dissipated in the window-frame kicker of Figure 4b become 4 times larger when the amplitude of the beam current increases from 1.5 [A] to 3.0 [A], as expected.

Table 1. The hysteresis and ohmic losses in various parts of the ferrite (columns 3-7) for the windowframe and C-type kickers for various amplitudes of the sinusoidal beam current ( ${ }^{\text {nd }}$ column)

\begin{tabular}{|c|c|c|c|c|c|c|}
\hline Model & $\begin{array}{c}\mathrm{I}_{\text {beam }} \\
{[\mathrm{A}]}\end{array}$ & $\begin{array}{c}\text { Total loss } \\
{[\mathrm{W} / \mathrm{mm}] 10^{-5}}\end{array}$ & $\begin{array}{c}\text { Ferrite loss } \\
{[\mathrm{W} / \mathrm{mm}] 10^{-5}}\end{array}$ & $\begin{array}{c}\text { Bus_Bar loss } \\
{[\mathrm{W} / \mathrm{mm}] 10^{-5}}\end{array}$ & $\begin{array}{c}\text { Copp-strip loss } \\
{[\mathrm{W} / \mathrm{mm}] 10^{-5}}\end{array}$ & $\begin{array}{c}\text { Vessel loss } \\
{[\mathrm{W} / \mathrm{mm}] 10^{-5}}\end{array}$ \\
\hline WF-Figure 4a & 1.5 & 29.62 & 11.50 & 1.95 & 15.4 & 0.75 \\
\hline WF-Figure 4b & 1.5 & 29.03 & 11.01 & 1.95 & 15.4 & 0.75 \\
\hline CM-Figure 7 & 1.5 & 7.51 & 1.76 & 2.85 & 0.58 & 4.66 \\
\hline WF-Figure 4b & 3.0 & 116.10 & 44.00 & 7.80 & 61.2 & 1.50 \\
\hline CM-Figure 7 & 3.0 & 30.00 & 7.03 & 11.39 & 2.30 & 9.3 \\
\hline
\end{tabular}

\section{The C-type kicker}

Although the copper strips inserted in the window-frame kicker reduce significantly the H_field generated by the circulating beam therefore the hysteresis losses in the ferrite are reduced significantly, the increase of the beam current by a factor of two will increase the hysteresis losses by a factor of four and the kicker may not be able to operate without cooling the kicker's ferrite. Line 5 in Table 1 shows that the hysteresis 
and eddy-current losses dissipated in the window-frame kicker of Figure $4 \mathrm{~b}$ become 4 times larger, when the amplitude of the beam current increases from 1.5 [A] to 3.0 [A]. In this section we present the results from the calculations on a C-type kicker magnet as an alternative RHIC abort kicker and we compare them with the two window-frame kickers. The cross section of the proposed C-type kicker is shown in Figure 7. The thin green ring is the stainless steel vacuum pipe and the blue shaded area corresponds to the ferrite. The aqua-marine rectangles are the current carrying conductors which are made of copper. The red circle in the center of the kicker shows the beam current which is simulated as a sinusoidal function with amplitude of 1.5 [A] and frequency 10 [MHz]. The thin brown area which surrounds the kicker is a 2 [mm] thick copper layer to further minimize the $\mathrm{H} \_$field induced by the beam current in the ferrite.

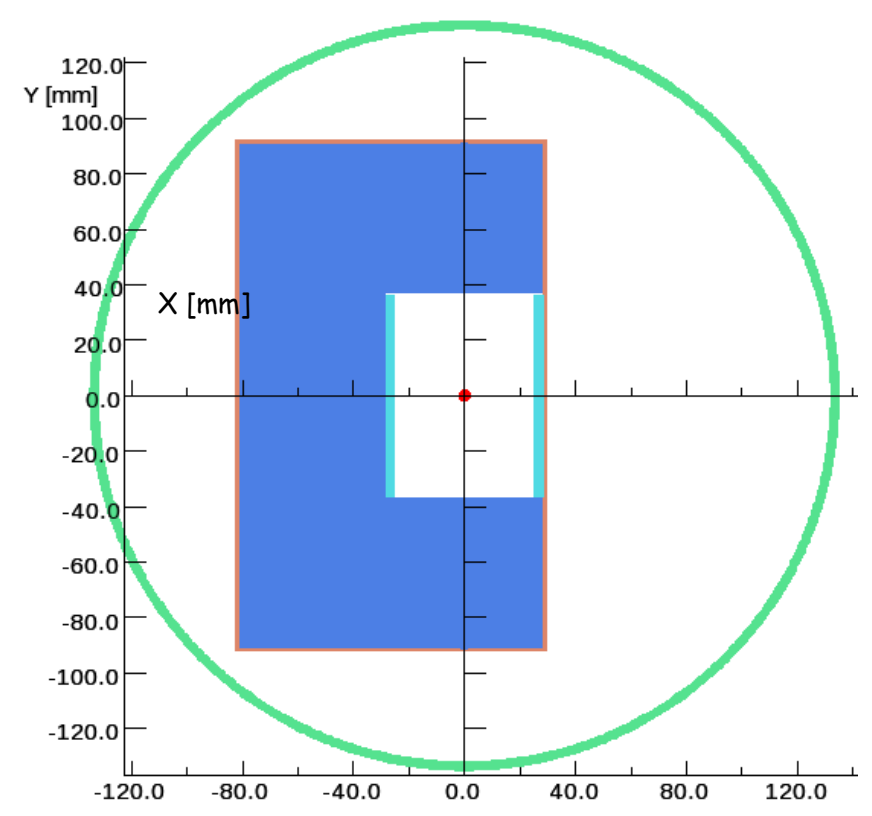

Figure 7. The cross section of the proposed C-type kicker. The thin green ring is the stainless steel vacuum pipe and the blue shaded area corresponds to the ferrite. The aqua-marine rectangles are the current carrying conductors which are made of copper. The red circle in the center of the kicker shows the beam current which is simulated as a sinusoid function with amplitude of 1.5 [A] and frequency 10 [MHz]. The thin brown area which surrounds the kicker is a 2 [mm] thick copper layer to further minimize the H_field induced by the beam current in the ferrite.

The physical properties of the materials used in the C-type kicker are identical to those used in the window-frame kickers. The yellow lines in Figure 8 correspond to the magnetic field lines as calculated by the 2D OPERA code. The results of the hysteresis and eddy-current losses from the 2D OPERA calculations are shown in line 4 of Table 1 . The comparison of the values in line 4 of Table 1 with those of lines 2 and 3 shows that the hysteresis and eddy-current losses in the materials of the C-type kicker are significantly lower than those of the window-frame kicker. The results from the calculations that use 3.0 [A] for beam current appear for comparison in lines 5 and 6 for the C-type and window-frame kickers respectively. 


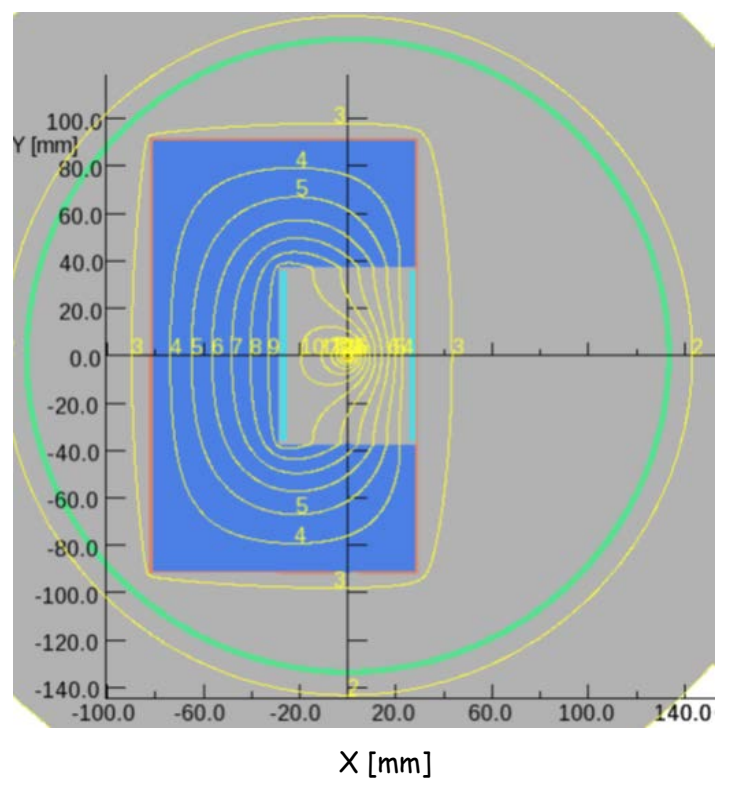

Figure 8. The yellow lines are the vector potential lines generated by the ac-module of the 2D OPERA computer model. Since the magnetic field lines must travel through the air-gap of the kicker magnet, the H_field in the ferrite is reduced appreciably. Further reduction of the H_field generated by the beam current in the ferrite is caused by the 2 [mm] copper-layer which surrounds the C-type kicker. The horizontal and vertical scales are in [mm].

\section{Transfer functions of window-frame and C-type kickers}

In this section we present the results from the calculations performed on the window-frame and C-type kickers to determine their transfer function. We use the "transient module" of the 2D OPERA computer code and we assume that the excitation current varies linearly in 100 nsec to the maximum value of 21 [kA]. Figure 9 shows the magnetic fields along the median plane of the window-frame (WF) and C-type (CM) kickers at the excitation current of 6300.0 [A]. At this excitation current the magnetic field of the Ctype kicker is $3.4 \%$ larger than that of the Window-frame kicker. This difference is partially due to the larger effective gap of the window-frame kicker by $2.0[\mathrm{~mm}](2.6 \%)$ to accommodate the insertion of the copper strip in the aperture of the kicker as shown in Figures 4a and 4b. Similar plot of the fields at the median plane of the kickers at excitation current of $21.0[\mathrm{kA}]$ is shown in Figure 10. At this excitation current the strength of the C-type kicker is still larger than that of the window-frame kicker. 


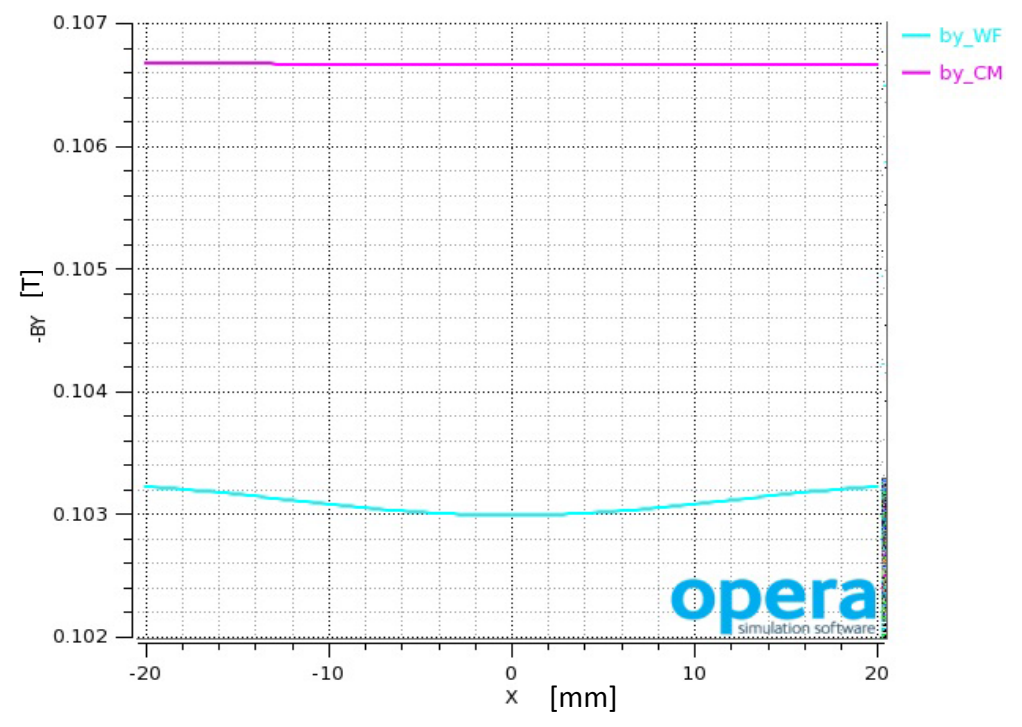

Figure 9. The magnetic field of the window-frame kicker (WF) and the C-type kicker (CM) along the median plane of the kicker at excitation current of 6300 [A]. This difference in the magnetic fields is due to the larger effective gap of the window-frame kicker by $2[\mathrm{~mm}](2.6 \%)$ to accommodate the insertion of the copper strip in the aperture of the kicker as shown in Figures 4a and $4 \mathrm{~b}$.

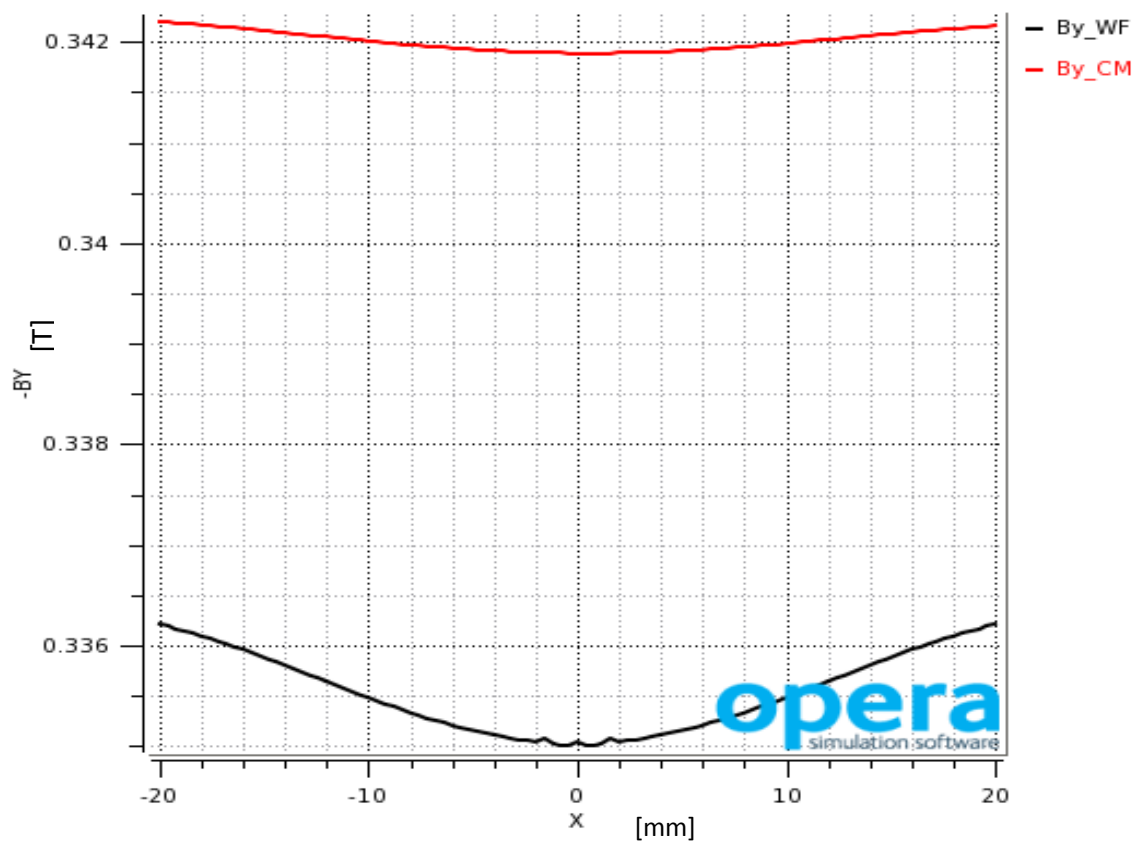

Figure 10. The magnetic field of the window-frame kicker (WF) and the C-type kicker (CM) along the median plane of the kicker at excitation current of 21.0 [kA]. This difference in the magnetic fields of the kickers is due to the larger effective gap of the window-frame kicker by 2 [mm] (2.6\%) to accommodate the insertion of the copper strip in the aperture of the kicker as shown in Figures 4a and 4b. 
Figure 11 is the plot of the magnetic field of the window-frame kicker shown in Figure 4b, and the magnetic field of the C-type kicker as a function of the excitation current. Both kickers are excited to a maximum current of 21.0 [kA] in $100 \mathrm{nsec}$, with a current varying linearly with time. One of the reasons the C-type kicker provides larger magnetic field than the window-frame kicker is that the gap of the window-frame kicker is by 2 [mm] larger to accommodate the copper strips.

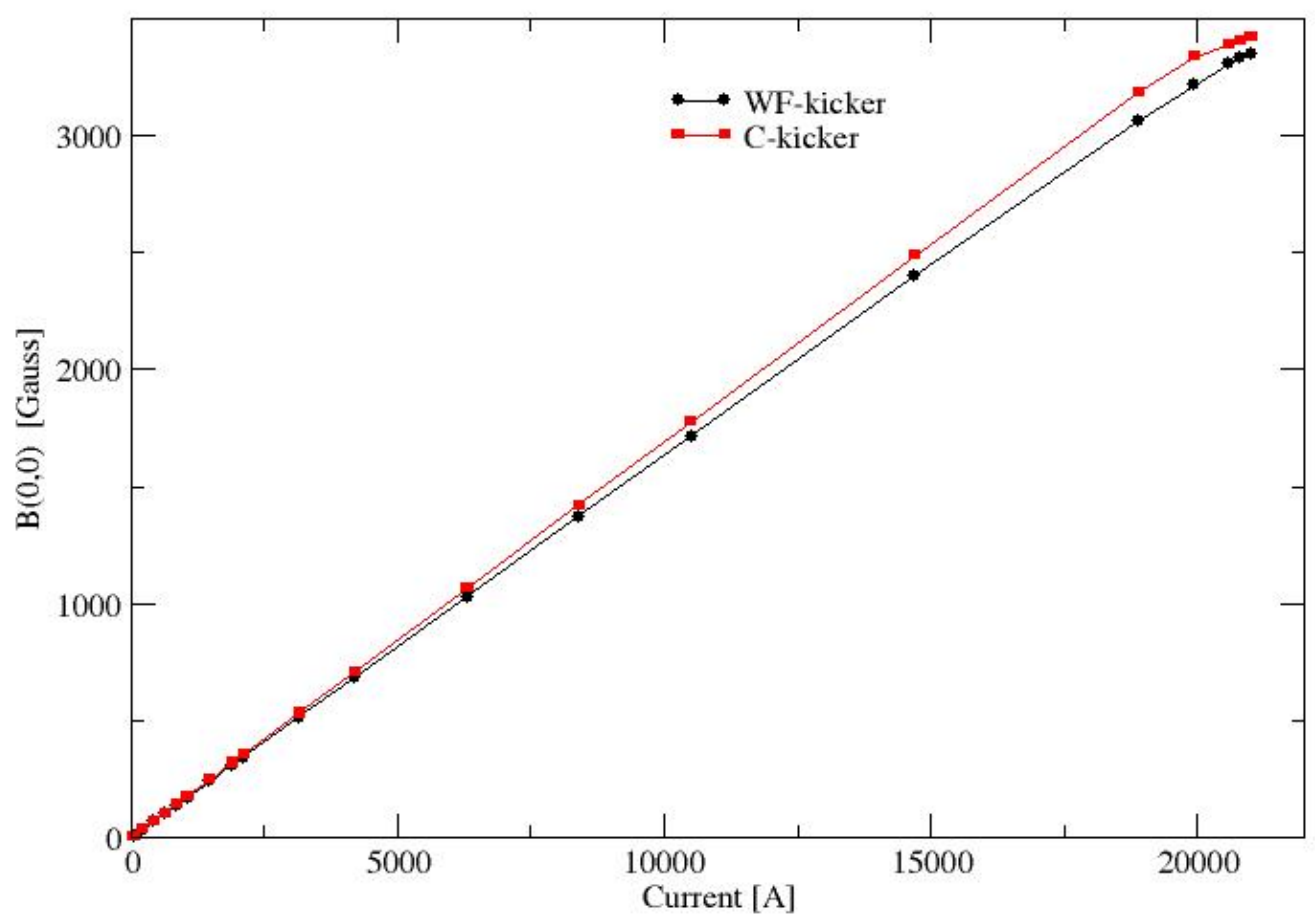

Figure 11. The black line is the plots of the magnetic field at $(x=0, y=0)$ of the window-frame kicker shown in Figure $4 \mathrm{~b}$, and the red trace if the magnetic field at $(\mathrm{x}=0, \mathrm{y}=0)$ of the C-type kicker, as a function of the excitation current. Both kickers are excited to the current of 21.0 [kA] in $100 \mathrm{nsec}$, with the current varying linearly with time.

Figure 12 is an expanded view of Figure 11 in the range of 19 to 21 [kA] which shows with the guidance of the black horizontal and vertical lines that the C-type kicker at 20.1 [kA] can generate the same field as the window-frame one at an excitation current of 21.0 [kA]. The percent difference of the magnetic field in the C-type kicker and the window-frame kicker is plotted in Figure 13 as a function of the excitation current. Although Figure 13 indicates that the C-type kicker is superior to the window-frame kicker because it produces a larger field at the same current, Figure 14 indicates that the inductance of the C-type kicker is larger by almost the same percentage, therefore the advantage of the C_type kicker is that it can generate the same field at $100 \mathrm{nsec}$ as the window-frame kicker with lower current of 20.1 [kA]. 


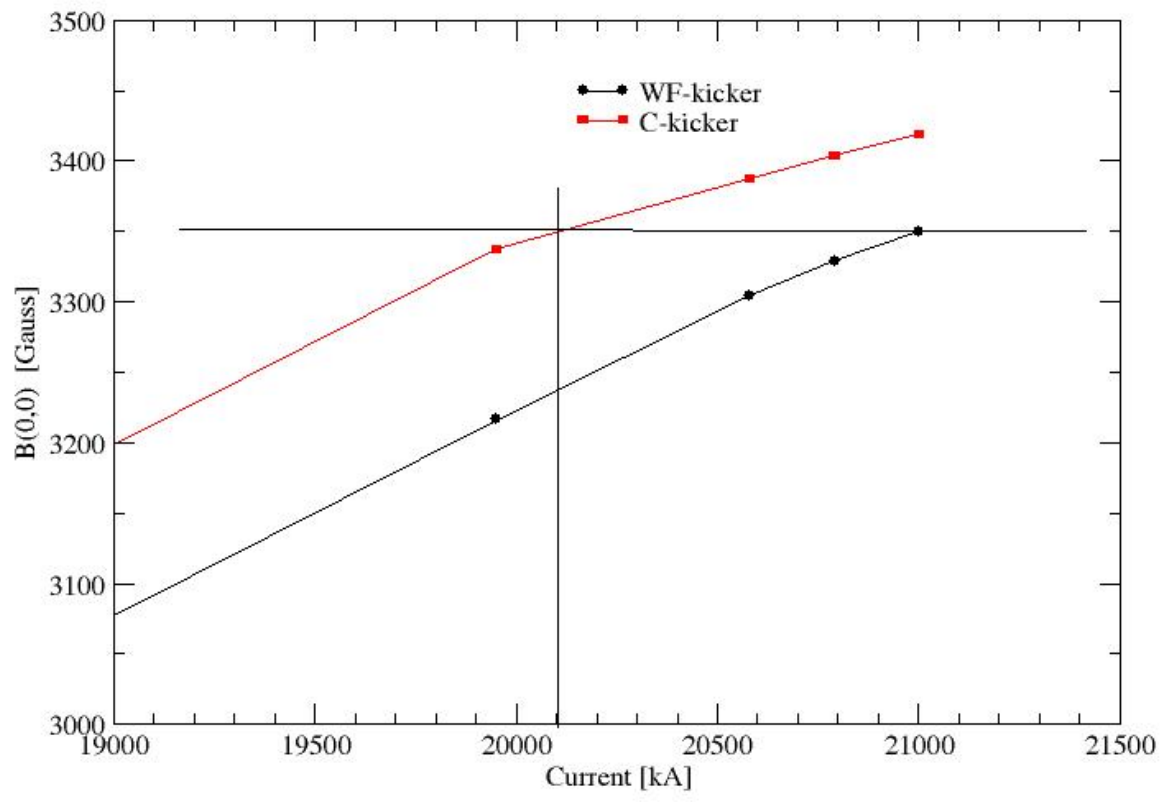

Figure 12. An expanded view of Figure 12 in the region of 19.0 to 21.0 [kA]. The horizontal and vertical black lines in the graph guides the eye to realize that the C-type kicker at 20.1 [kA] can generate the same field as the window-frame one at an excitation current of 21.0 [kA].

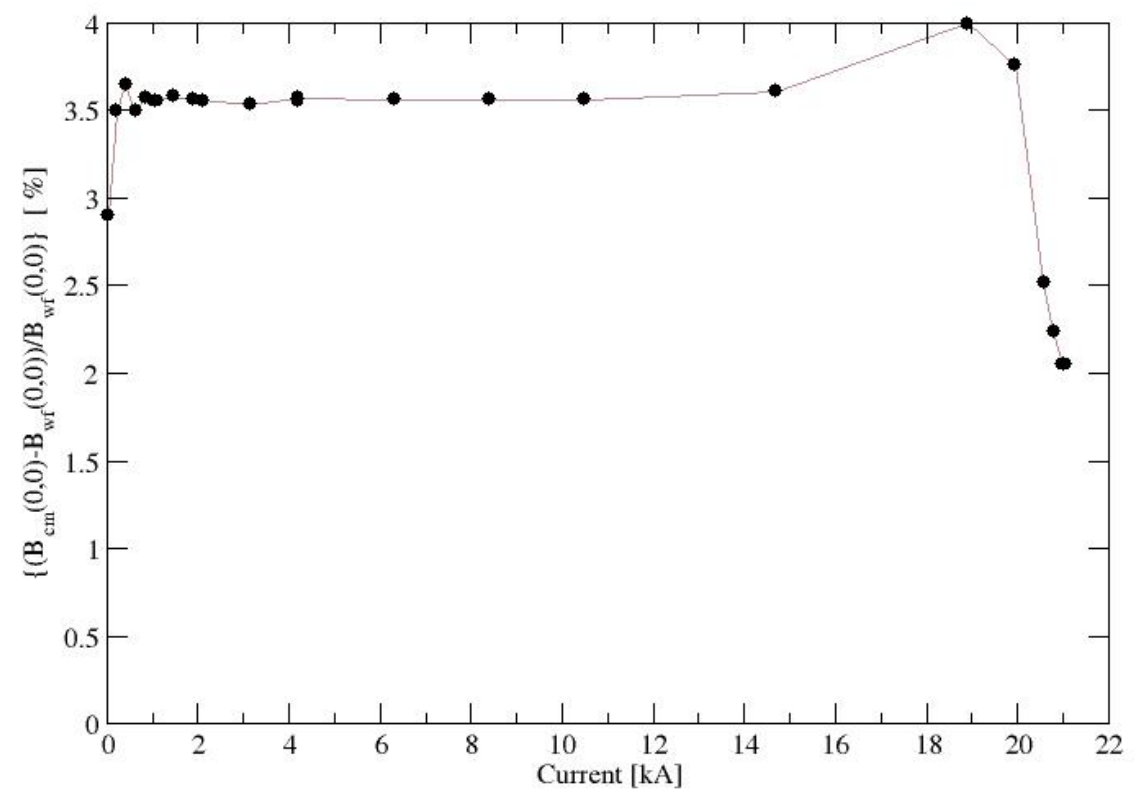

Figure 13. The percent difference of the magnetic field in the C-type and window-frame kickers as a function of the excitation current. 


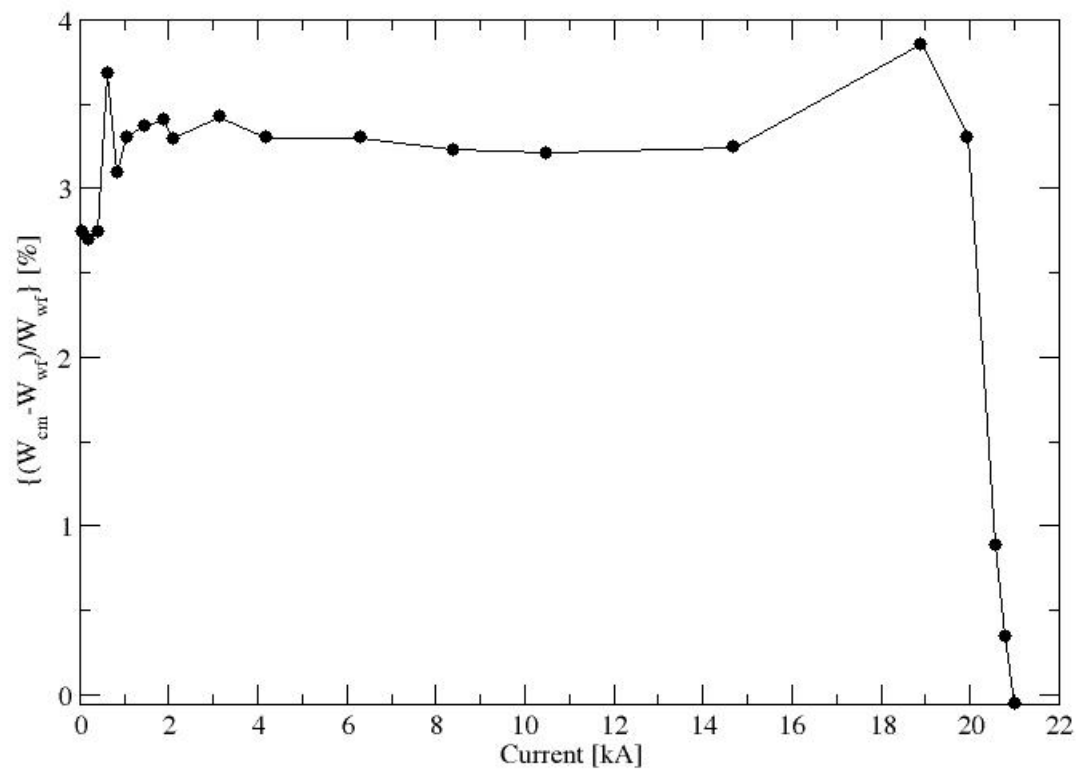

Figure 14. The percent difference between of the total energy stored in the C_type and window-frame kickers. This percent difference in the energies stored is the same as the percent difference between the Inductances of the kickers.

\section{Conclusions}

Calculations performed on a window-frame and a C-type kicker using the "ac" module of the 2D OPERA computer code show that the hysteresis losses in the ferrite due to the H_field generated by the circulating beam bunches are significantly lower in the C-type kicker as can be seen from the values in Table 1 . Also the field generated by the excitation current of the kicker is $\sim 3.4 \%$ higher for the C-type kicker as compared to the window-frame kicker. However this apparent advantage of the C-type kicker vs the window-frame one is compensated by the increased inductance of the C-type kicker as shown in Figure 14. Thus the C-type kicker can generate in 100 nsec at 20.1 [kA] the same maximum field as the windowframe kicker does at 21.0 [kA]. The hysteresis losses in the ferrite of the C-type kicker for a 3.0 [A] pick sinusoidal current are lower than the corresponding losses of the window-frame kicker with peak beam current of 1.5 [A]. We also have included an Appendix where we describe a method which may further reduce the hysteresis losses of the window-frame kicker.

\section{Appendix A1}

In this appendix we describe a method that can be used to further minimize the hysteresis losses in the ferrite of the window-frame kicker. As we mentioned in one of the earlier sections, the reduction of the hysteresis losses can be achieved by reducing the $\mathrm{H}$ _field generated by the circulating beam bunches in the ferrite. Figure A1.1 is a horizontal cross section of the kicker at the median plane of the kicker. The dark rectangles are the two sides of the kicker made of ferrite and the aqua-marine rectangles are the current caring conductors of the kicker. The green arrow shows the beam direction. The direction of the H_field generated in the ferrite by the beam current is shown on the ferrite by the appropriate circles. 


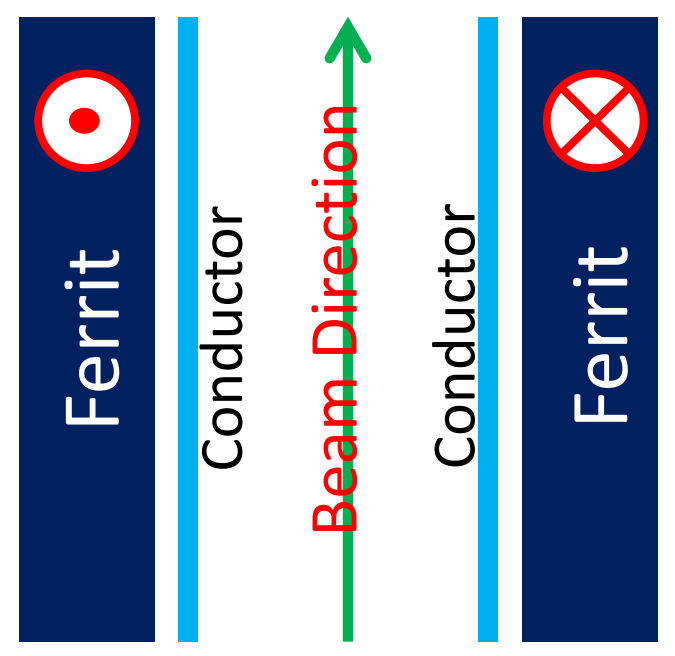

Figure A1.1. Cross section of the window-frame RHIC-abort kicker at the horizontal median plane. The dark rectangles are the vertical sides of the ferrite, the aqua-marine rectangular bars are part of the conductors and the green arrow shows the beam direction. The direction of the H_field generated in the ferrite by the beam current is shown on the ferrite by the appropriate circles.

With the figures that follow below we describe the proposed method to minimize further the H-field generated by the circulating beam in the ferrite. We start with the figure A1.2 which is the same as the figure A1.1 except that the we cover the vertical sides of the kiker's ferrite with a 1 [mm] thin copper sheet as shown by the red lines in Figure A1.2.
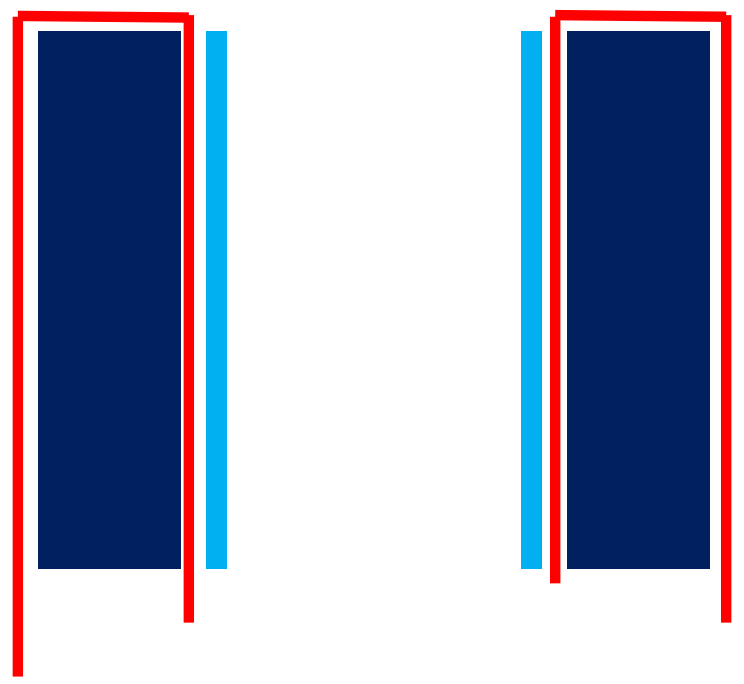

Figure A1.2 The thin red lines are $\sim 1[\mathrm{~mm}]$ thin copper sheets which cover the vertical sides of the kicker's ferrite which is shown by the dark blue rectangle. 
Subsequently we connet the thin copper sheets with copper wires as shown with the thick dark-red lines in Figure A1.3.

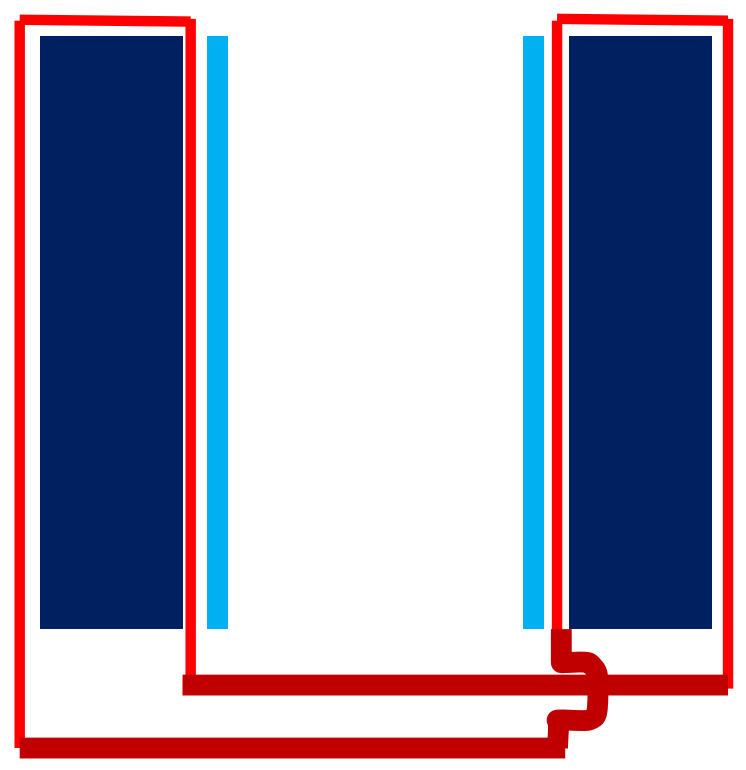

Figure A1.3 The thin copper sheets covering the ferrite (thin red lines) are connected together with wires (thick dark red lines).

As we mentioned earlier the beam current (green arrow in Figure A1.4) generates in the ferrite an H_field whose direction is indicated in Figure A1.4 by the red circles in the ferrite material. Now the change of the magnetic flux generated by the B_field in the ferrite material induces eddy currents in the current sheets shown by the thin red lines. The direction of the eddy currents in the copper sheets and the copper wires is shown by the small green arrows. These eddy currents generate in the ferrite material an H_field (green circles in the ferrite) which dents to cancel the H_field (red circles) generated by the beam.

By connecting the copper sheets the way it is shown in Figure A1.4 we may reduce further the H_field generated by the circulating beam current, therefore reduce further the hysteresis losses in the ferrite. Although the copper sheets are beneficial in reducing the $\mathrm{H}$ _field in the ferrite, one may ask on the effect of the copper sheets on the magnetic field generated by the excitation current in the ferrite. The answer to this question is provided by examining Figure A1.5, which shows the direction of the excitation current as purple arrows in the conductors. The excitation current generates in the ferrite a magnetic field whose direction is shown by the red circles in Figure A1.5. The change of the magnetic flux in the ferrite generates eddy-current in the copper sheets and the wires. The direction of these currents is shown by green arrows in Figure A1.5. The direction of the eddy-currents of one loop is such that it cancels the eddy currents of the other loop therefore no magnetic field is generated by the eddy currents of copper loops. 


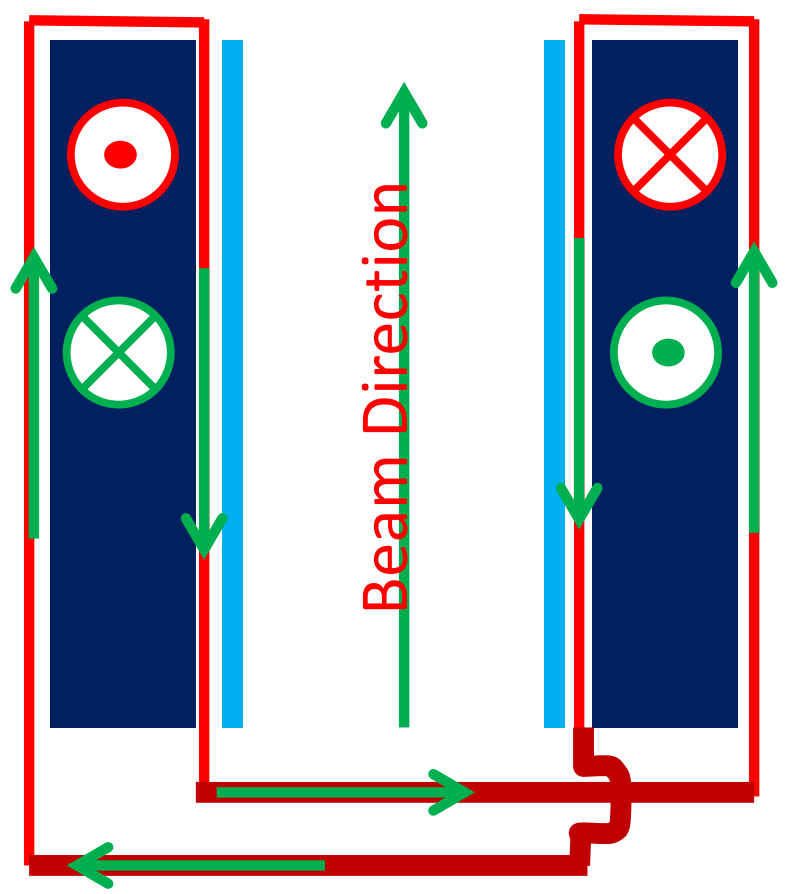

Figure A1.4. The change of the magnetic flux in the ferrite generates eddy-currents induced on the copper sheets (thin red lines) and the copper wires (thick dark lines). These eddy currents generate an H_field in the ferrite (green circles) which tends to cancel the H_field (red circles) generated by the beam current.

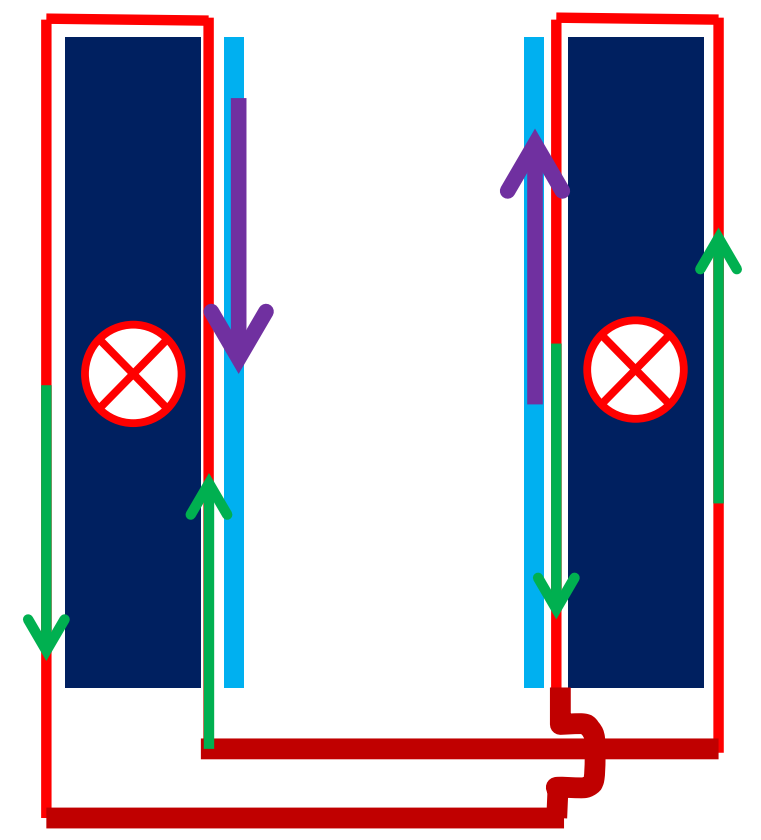

Figure A1.5. This figure helps understand the effect of the copper sheets and wires (red lines) on the magnetic field generated by the excitation current (purple arrows) in the conductors. The green arrows are the eddy currents generated by the change of the magnetic flux in the ferrite. The direction of the eddy currents in each copper loop is such that they cancel each other therefore no magnetic field is generated by the eddy currents generated in the copper sheets. 